\section{Influência da música em variáveis psicofisiológicas durante um exercício submáximo em ciclossimulador}

\section{Music influence on psychophysiological variables during submaximal exercise in cycle simulator}

Marcelo Bigliassi ${ }^{1}$

Elisa J. Santos ${ }^{1}$

Thiago Ferreira Dias Kanthack ${ }^{1}$

Henrique Bortolotti ${ }^{1}$

Leandro Ricardo Altimari ${ }^{1}$

\section{Resumo}

O Colégio Americano de Medicina do Esporte (CAME) propõe algumas recomendações para a prática de atividade física na tentativa de aprimorar a saúde ou prevenir problemas crônicos degenerativos ao ser humano, dessa forma algumas estratégias ergogênicas podem auxiliar essa prática, entre elas o uso da música, capaz de possibilitar um melhor desempenho e menor percepção de esforço. Sendo assim, o objetivo deste estudo foi investigar o efeito da música em variáveis psicofisiológicas de adultos durante a prática de atividade física prescrita segundo as recomendações do CAME/AAC, (2007). Participaram deste estudo 10 voluntários saudáveis do sexo masculino $(22,3 \pm 1,8$ anos, $175,0 \pm 5,5 \mathrm{~cm}$ e $74,8 \pm 11,0 \mathrm{Kg})$ que realizaram duas sessões experimentais de 30 minutos em ciclossimulador com intensidade correspondente a $60 \%$ da frequência cardíaca de reserva $\left(\mathrm{FC}_{\text {Limiar de Treino }}\right)$, sobre duas condições: Música $(\mathrm{M})$ e Controle (C). Foram avaliadas as variáveis: potência média $\left(\mathrm{W}_{\mathrm{MED}}\right)$, variabilidade da frequência cardíaca (VFC), percepção subjetiva de esforço (PSE) e o estado de humor (EH) dos participantes. Para as variáveis: PSE, $\mathrm{W}_{\mathrm{MED}}$, VFC foi usado o teste de análise de variância (ANOVA - two-way) para medidas repetidas, seguido do post-hoc de Bonferroni e para a variável EH foi usado o teste de Wilcoxon. O nível de significância estatística adotada nas análises foi de $5 \%$. Nenhuma diferença estatisticamente significante foi encontrada para as variáveis $\mathrm{W}_{\mathrm{MED}}$, VFC, PSE e EH dos participantes entre as condições $\mathrm{Me} \mathrm{C}(P>0,05)$. Conclui-se que a música não foi capaz de influenciar as medidas psicofisiológicas em esforço submáximo.

\section{Palavras-chave}

Atividade Motora; Música; Frequência Cardíaca.

\begin{abstract}
The American College of Sports Medicine and the American Heart Association (ACSM and AHA) bring some recommendations for the practice of physical activity with the intention of health increase or prevent diseases like degenerative problems on human, in this way some ergogenics strategies can help this practice, between them, the use of music, capable to enable a better performance and lower the perception of effort (RPE). Thus, the objective of this study was to investigate the effect of music in psychophysiological variables in adults during practice of physical activity following the recommendations of ACSM/AHA. Participated of this study 10 healthy volunteers from masculine genre $(22.3 \pm$ 1.8 years, $175.0 \pm 5.5 \mathrm{~cm}$ and $74.8 \pm 11.0 \mathrm{Kg}$ ) that did two experimental session with 30 minutes in a cyclesimulator with intensity corresponding to $60 \%$ of the heart reserve rate ( $H R$ training threshold $)$, in two conditions: with music (M) and control (C). Was evaluated the variables: power output (WMED), heart rate variability (HRV), rate perception of effort (RPE) and mood status (MS) of volunteers. For the variables RPE, WMED, HRV was used the variance analyses test (ANOVA - two way) for repeated measures followed by the post hoc of Bonforreoni and the Wilcoxon's test was used to compare the $M E$. The level of statistical significance was $5 \%$. No differences were found in any variables WMED, $H R V, R P E$ e MS of participants between conditions $M$ and $C(P>0.05)$. Concluded that music don't was capable to change psychophysiological measures in submaximal effort.
\end{abstract}

\section{Keywords}

Motor Activity; Music; Heart Rate.
Rev Bras Ativ Fis Saúde p. 532-542 DOI: http://dx.doi.org/10.12820/23171634.2012v17n6p532

1 Grupo de estudo e pesquisa em sistema neuromuscular e exercício, Centro de Educação Física e Esporte, Universidade Estadual de Londrina, PR, Brasil 


\section{INTRODUÇÃO}

Dados de países desenvolvidos indicam que menos de $15 \%$ da população adulta pratica exercícios físicos regularmente ${ }^{1}$. Reichert et al. ${ }^{2}$, tem mostrado que indivíduos tendem a criar barreiras para a prática de atividade física, sendo alguns dos motivos mais citados: falta de dinheiro, cansaço, falta de companhia e falta de tempo ${ }^{2}$. Tendo isso em vista, alguns posicionamentos foram publicados com intuito de nortear a prescrição de exercícios para prevenção de doenças e manutenção da saúde, por exemplo, o Colégio Americano de Medicina do Esporte e a Associação Americana do Coração, que publicaram em 2007 recomendações para práticas de atividades físicas em adultos saudáveis, estabelecendo que indivíduos adultos devam acumular 30 minutos de atividades aeróbicas com intensidade moderada por pelo menos cinco dias semanais ou 20 minutos de intensidade vigorosa pelo menos três dias semanais, a combinação de atividades moderadas e vigorosas também é aceita nessas recomendações. Sendo que a intensidade proposta pode variar de 55 a $90 \%$ da frequência cardíaca (FC) máxima (FCmax), ou de 40 a $85 \%$ da frequência cardíaca (FC) de reserva (FCres) ${ }^{3}$.

Conhecendo os benefícios da relação atividade física e saúde, novos meios que potencializem ou estimulem essa prática se fazem necessários. Recentes estudos têm utilizado a música como um meio capaz de atender a essa demanda, apresentando resultados psicofisiológicos expressivos, em exercícios de caráter máximo e submáximos ${ }^{4-8}$. Seus efeitos podem alterar padrões comportamentais como o estado de humor e a sensação de esforço, modificando de forma indireta respostas hormonais e metabólicas ${ }^{9-11}$. Sua aplicação pode decorrer em paralelo ao exercício, modificando respostas autonômicas e perceptuais, favorecendo e acelerando o processo de recuperação principalmente em tarefas de menor intensidade ${ }^{12}$. Assim o presente estudo teve como objetivo comparar o efeito da música em variáveis psicofisiológicas com a condição isenta de intervenção, seguindo as recomendações do CAME/AAC, (2007) para prática de atividade física para adultos.

\section{MÉTODOS}

\section{Amostra}

Participaram deste estudo 10 voluntários saudáveis do sexo masculino, alunos do curso de Educação Física da Universidade local $(22,3 \pm 1,8$ anos, 175,0 \pm 5,5 cm e $74,8 \pm 11,0 \mathrm{Kg}$ ), os quais não apresentavam histórico de lesões ou tabagismo nos seis meses anteriores ao experimento, para todos os sujeitos foram esclarecidos os procedimentos do estudo ao qual seriam submetidos e permitido que deixassem de participar do mesmo a qualquer momento, em seguida assinaram um termo de consentimento livre e esclarecido. Eram sujeitos fisicamente ativos (pelo menos $3 \mathrm{x} /$ semana) que praticavam diferentes modalidades esportivas recreacionais ou qualquer atividade física por pelo menos 6 meses. Ressalta-se que todos os sujeitos envolvidos no presente estudo relataram não fazer uso de nenhum tipo de medicamento. Previamente ao experimento, os voluntários foram instruídos sobre a necessidade de abstinência a qualquer atividade física vigorosa e ingestão substâncias cafeinadas ou alcoólicas nas 24 horas precedentes às sessões de teste. Além disso, realizaram os testes sempre no mesmo período do dia $( \pm 1 \mathrm{~h})$, visando evitar variações circadianas. Este estudo foi aprovado pelo Comitê de Ética e Pesquisa da Universidade Estadual de Londrina segundo a resolução 196/96 (protocolo 212/2011). 


\section{Delineamento experimental}

Inicialmente os voluntários compareceram ao laboratório para mensurações antropométricas e realização de teste pré- experimental com o propósito de familiarização dos sujeitos com os equipamentos e procedimentos experimentais os quais seriam submetidos. Posteriormente, todos os voluntários retornaram para realizar duas sessões de teste em um ciclossimulador $\left(\right.$ Velotron $^{\mathrm{TM}}$, Dynafit Model, Racer Mate ${ }^{\circledast}, \mathrm{USA}$ ), com um intervalo mínimo de 48 horas e maximo de 168 horas entre as sessões, onde cada sessão tinha uma duração de 30 minutos em intensidade submáxima personalizada calculada pelo método de frequência de reserva 12 , segundo a fórmula abaixo:

$$
\mathrm{FC}_{\text {Limiar de Treino }}=(\mathrm{FCmax}-\mathrm{FCrepouso}) * 60 \%+\mathrm{FCrepouso}
$$

Onde FCmax foi calculada conforme sugere Tanaka et al., (2001) ${ }^{13}$.

Vale ressaltar que, estudos tem considerado essa equação valida em crianças e adolescentes ${ }^{13,14}$, e adultos jovens ${ }^{13,15,16}$, por apresentar resultados preditivos bastante próximos da FCmax. Já a FCrepouso foi definida como a média do minuto final de um período de 10 min em repouso na posição sentada. Durante todo o tempo o sujeito ficava sozinho no ambiente laboratorial sem influencia de qualquer estimulo auditivo ou visual.

Após a obtenção da variabilidade da frequência cardíaca (VFC) e a avaliação do estado de humor pré-exercício, com procedimentos descritos nos itens a seguir, os voluntários foram posicionados no ciclossimulador para início do teste. Vale ressaltar que a frequência cardíaca serviu como parâmetro de controle da intensidade do exercício. $\mathrm{O}$ equipamento possui regulagens ajustáveis que permitiram adequar às dimensões de acordo com as medidas antropométricas dos voluntários. Antes do início do primeiro teste, após devidamente posicionado, o voluntário recebia instruções sobre o funcionamento da troca de relação de engrenagens do ciclossimulador, a posição sentada a ser mantida durante todo o teste, sobre como responder a percepção subjetiva de esforço (PSE), a faixa de rotação por minuto (RPM) a ser mantida $(70-110 \mathrm{RPM})$ e a intensidade do teste $\left(\mathrm{FC}_{\text {Limiar de Treino }}\right)$. Sempre que a FC desviava-se $10 \%$ acima do valor de $\mathrm{FC}_{\text {Limiar de Treino, }}$ o voluntário era instruído a trocar a relação de engrenagens para uma mais leve visando voltar à intensidade estipulada. Após estas informações, os voluntários realizavam cinco minutos de aquecimento com carga leve e de livre escolha, e então iniciava a sessão de teste.

Todos os voluntários realizaram os testes em duas condições em dias separados, sendo uma com a presença de música $(\mathrm{M})$ e outro controle $(\mathrm{C})$ com ausência desta, com ordem aleatorizada. A sequência de 30 minutos de música foi escolhida pelo voluntário permitindo assim que eles escolhessem as músicas que os incentivassem a fazer exercícios, uma vez que a preferência musical é um fator importante no desempenho ${ }^{17}$. A música era transmitida através de um aparelho mp4 (Sony ${ }^{\circ}$ NWZ-E473) a um fone direto no ouvido do sujeito.

Em ambas as condições, a cada dois minutos de teste foram coletadas a potência média do período $\left(\mathrm{W}_{\mathrm{MED}}\right)$ e a PSE. Após o fim dos 30 minutos de exercício, foram realizadas as avaliações da VFC de recuperação pós-exercício e uma nova avaliação do estado de humor. A variável temperatura ambiente foi mantida entre 21 e $24^{\circ} \mathrm{C}$ para todas as coletas. Todos os procedimentos são descritos nos itens subsequentes. 


\section{Variabilidade da Frequência Cardíaca (VFC)}

Esta variável foi obtida nos momentos pré- e pós-exercício com a intenção de mensurar o estresse autonômico gerado pelo exercício. Imediatamente após a chegada do voluntário ao laboratório, ocorria a colocação do cardiofrequencímetro (POLAR $^{\oplus}$ RS800 CX, Finlândia). Este equipamento permite o monitoramento da frequência cardíaca batimento a batimento pela detecção das ondas $\mathrm{R}$ do ciclo cardíaco com validação na literatura ${ }^{18}$. Após aplicação de gel condutor, o sensor foi posicionado conforme orientações do fabricante sobre o tórax do voluntário. Realizado este procedimento, iniciava-se a coleta da VFC pelo período de dez minutos, com o voluntário confortavelmente sentado, em silêncio e sem realizar qualquer movimento brusco.

Os últimos cinco minutos de repouso foram utilizados para análise da VFC. Os registros dos intervalos $\mathrm{R}-\mathrm{R}$ foram filtrados para eliminar possíveis ruídos provenientes de batimentos ectópicos ou erros de leitura do aparelho na ordem de 20 bpm, sendo que o percentual de correção dos intervalos R-R não ultrapassou $2 \%$. O cálculo dos parâmetros no domínio do tempo foi realizado a partir dos seguintes índices: raiz quadrada da média das diferenças sucessivas ao quadrado entre intervalos R-R adjacentes (RMSSD) e o desvio padrão dos intervalos R-R normais (SDNN). O RMSSD é considerado refletir a atividade parassimpática e o SDNN como indicador global da atividade do sistema nervoso autônomo (SNA) pela VFC ${ }^{18}$. A porcentagem de intervalos $\mathrm{RR}$ adjacentes com diferença de duração maior que $50 \mathrm{~ms}$ (pNN50), que também parece refletir a atividade parassimpática.

A transformação rápida de Fourier (FFT) foi usada para estimar a densidade do espetro de potência da variabilidade dos intervalos R-R. A densidade do espectro de potência foi quantificada pela medida da área sob duas bandas de frequência: componente de baixa frequência $(\mathrm{BF})$, equivalente a 0,04 a $0,15 \mathrm{~Hz}$, o qual parece refletir a atividade de ambos os ramos do SNA e componente de alta frequência (AF), equivalente a 0,15 a $0,4 \mathrm{~Hz}$, assumido como indicador da atividade autonômica parassimpática. Tanto LF quanto HF foram expressos em valores absolutos $\left(\mathrm{ms}^{2}\right)$ e em unidades normalizadas (un). Os dados em unidades normalizadas (BFun e AFun) foram utilizados com a intenção de diminuir o efeito da variação interindividual nas escalas absolutas $\left(\mathrm{ms}^{2}\right)$, sendo obtidos a partir da equação $\left\{[(\mathrm{AF} \text { ou } \mathrm{BF}) /(\mathrm{AF}+\mathrm{BF})]^{*} 100\right\}$. O balanço simpato-vagal foi expresso pela razão entre eles (razão $\mathrm{BF} / \mathrm{AF}$ ) ${ }^{18}$. A determinação dos componentes espectrais e temporais foi realizada por meio do HRV Kubios Software ${ }^{\circledR}$ (Biosignal Laboratory, University of Kuopio, Finlândia).

\section{VFC de recuperação e reativação parassimpática pós-exercício.}

Em ambas as condições, após o final da sessão de exercício, avaliaram-se a dinâmica de recuperação da VFC pelo período de 10 minutos. A cada período de 30 segundos foram calculadas as variáveis RMSSD, o desvio padrão da dispersão perpendicular à linha identidade (SD1) e o desvio padrão da dispersão ao longo da linha identidade (SD2) dos intervalos RR projetados no gráfico de Poicaré ${ }^{19}$. Estas duas últimas representam respectivamente o efeito da alteração da atividade parassimpática e a interação entre alterações simpática e parassimpática sobre a VFC ${ }^{20}$.

\section{Avaliação do Estado de Humor}

Finalizado o procedimento de VFC, os voluntários respondiam o Brunel Mood Scale (BRUMS), que corresponde a um questionário desenvolvido para permitir uma rápida mensuração do estado de humor, e suas alterações ${ }^{21}$, de populações 
compostas por adultos e adolescentes. Este é composto por 24 questões que posteriormente são estratificadas em seis domínios de indicadores de humor (raiva, confusão, depressão, fadiga, tensão e vigor) ${ }^{22}$, com versão traduzida e validada no Brasil ${ }^{23}$. Após o fim dos 30 minutos de exercício, os voluntários também respondiam novamente o questionário, visando verificar as alterações de humor entre o início e fim do experimento devido à aplicação da carga de treinamento ${ }^{24}$.

\section{Percepção Subjetiva de Esforço (PSE)}

A PSE foi usada para avaliar o efeito do exercício sobre as variáveis psicofisiológicas durante a atividade. Para isto, a cada dois minutos de teste, os voluntários foram instruídos a relatarem sua percepção subjetiva de esforço (PSE) de acordo com a escala de 15 pontos $(6-20)$ de Borg, (1982) ${ }^{25}$, que estava visível à frente do sujeito, respondendo a questão: "o quão intenso está à tarefa neste momento?" 26,27. Para todos os experimentos os valores para percepção extremamente fácil (7) e o valor extremamente difícil (19) serviram para de âncora para instrução dos sujeitos ${ }^{28}$. Também houve a observação da importância da veracidade da PSE informada, realçando que o fato de responder um valor inferior ou superior em relação a real percebida não influenciaria na avaliação de rendimento. A PSE exerceu a função de resposta perceptual ao exercício, uma vez que mantida a carga fisiológica, estímulos auditivos externos poderiam influenciar a resposta subjetiva a um mesmo esforço.

\section{Percepção Subjetiva de Esforço da sessão (PSE da sessão)}

A PSE da sessão foi utilizada como ferramenta para quantificar a carga de treinamento da sessão em ambas às condições, utilizando-se da escala Category Ratio (CR 10) (0 - 10) de Borg (1982) ${ }^{25}$, após 15 minutos do final da sessão de teste onde os voluntários foram instruídos a responder a questão: "o quão difícil foi para você realizar a sessão de treinamento?". Os valores para percepção extremamente fácil (1) e o valor extremamente difícil (9) serviram para de âncora para instrução dos voluntários, que podiam responder qualquer da escala a cada meio ponto, ou seja, eram aceitos valores decimais (ex: 6,5). Este valor obtido foi multiplicado pelo tempo em exercício (30 min.) e definido como o valor de carga interna da sessão em unidades arbitrárias.

\section{Procedimentos Estatísticos}

Finalizado o experimento, os dados foram submetidos à estatística descritiva e ao teste de Shapiro-Wilk para verificar se os dados apresentavam distribuição normal. As variáveis que não apresentaram distribuição normal (o delta de variação do R-Rmédio e HF) foram transformadas em logaritmo natural possibilitando a aplicação de estatística paramétrica. Os dados de PSE, $\mathrm{W}_{\mathrm{MED}}$, VFC e variáveis da avaliação dinâmica de recuperação da VFC foram submetidos ao teste de esfericidade de Mauchly e seguidos da análise de variância (ANOVA) fatorial para medidas repetidas, com teste de Bonferroni como procedimento post-hoc. Os dados de taxa linear de aumento da PSE (Slope), obtida por meio de regressão linear dos dados desta variável, seus respectivos coeficientes de explicação, os dados de PSE da sessão e os dados do instrumento de BRUMs foram submetidos ao teste não-paramétrico de Wilcoxon. O Delta de variação da VFC entre repouso e recuperação foi submetido ao teste " $t$ " de Student para variáveis dependentes. A significância adotada foi de $p<0,05$. 


\section{RESULTADOS}

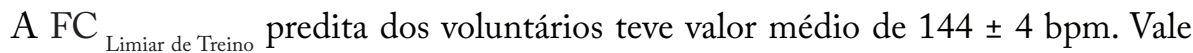
ressaltar que não houve diferença significante entre a $\mathrm{FC}_{\text {Limiar de Treino }}$ média predita e a obtida nas condições $\mathrm{C}$ e $\mathrm{M}\left(\mathrm{ICC}=0,904 ; \mathrm{CI}_{95 \% \mathrm{ICC}}(0,727-0,974)\right.$ e $\mathrm{W}_{\text {MED }}$ $\left(F=1.045, P=0.396, R^{2}=0.061\right)$. Quanto aos valores da $\mathrm{W}_{\mathrm{MED}}$ dos 30 minutos de teste, não se constatou diferenças significantes entre as condições C e M (106,3 \pm $13,3 \mathrm{~W}$ vs 107,0 $\pm 9,3 \mathrm{~W}$, respectivamente; $P>0,05$ ).

A PSE aumentou significativamente à medida que o tempo em teste aumentava $\left(F=14.718, P<0.001, R^{2}=0.45\right)$, apesar da intensidade constante do exercício. Porém, semelhante ao comportamento da $\mathrm{W}_{\text {MED }}$ (Figura 1), a dinâmica da PSE também não apresentou diferença significante entre as condições $(F=0.064, P=$ $\left.0.946, R^{2}=0.004\right)$, conforme demonstrado na Figura 2 , com o mesmo contexto para a taxa de inclinação (SLOPE) $(P>0,05)$ e coeficiente de explicação $\left(R^{2}\right)(P>$ $0,05)$ desta variável. Adicionalmente, não houve diferença na carga interna percebida pelos voluntários, representada pela PSE da sessão $(P>0,05)$.

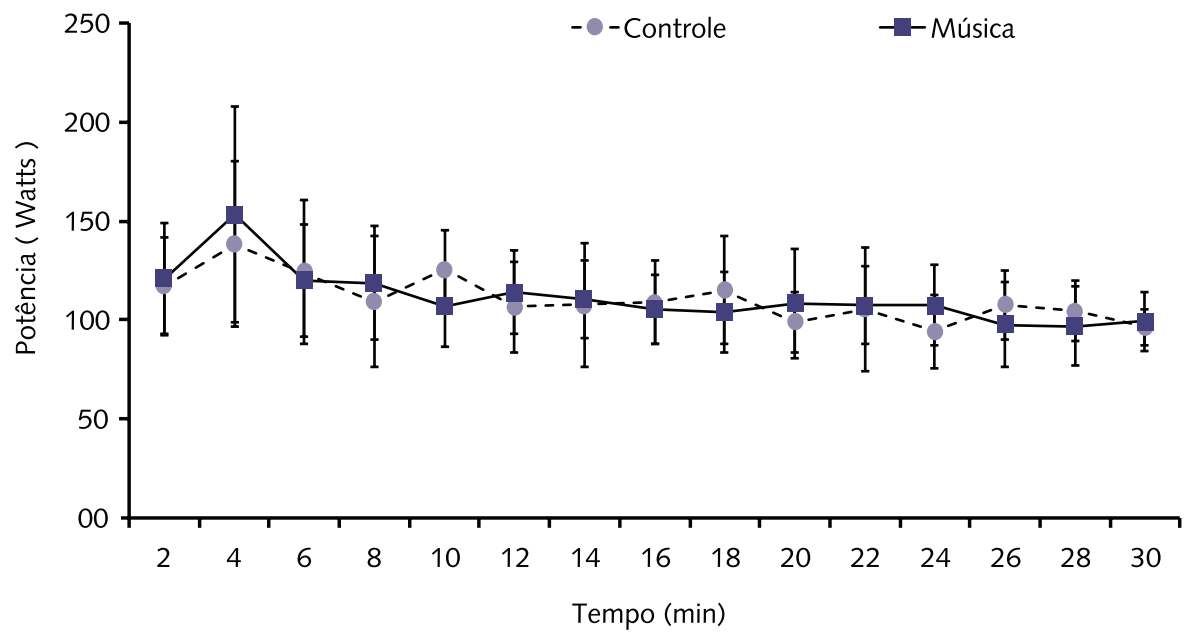

Figura 1 - Comportamento da potência média a cada dois minutos ao longo do teste de 30 minutos nas condições Controle e Música, expressos sob a forma média (marcadores) e desvio padrão (barra de erros).

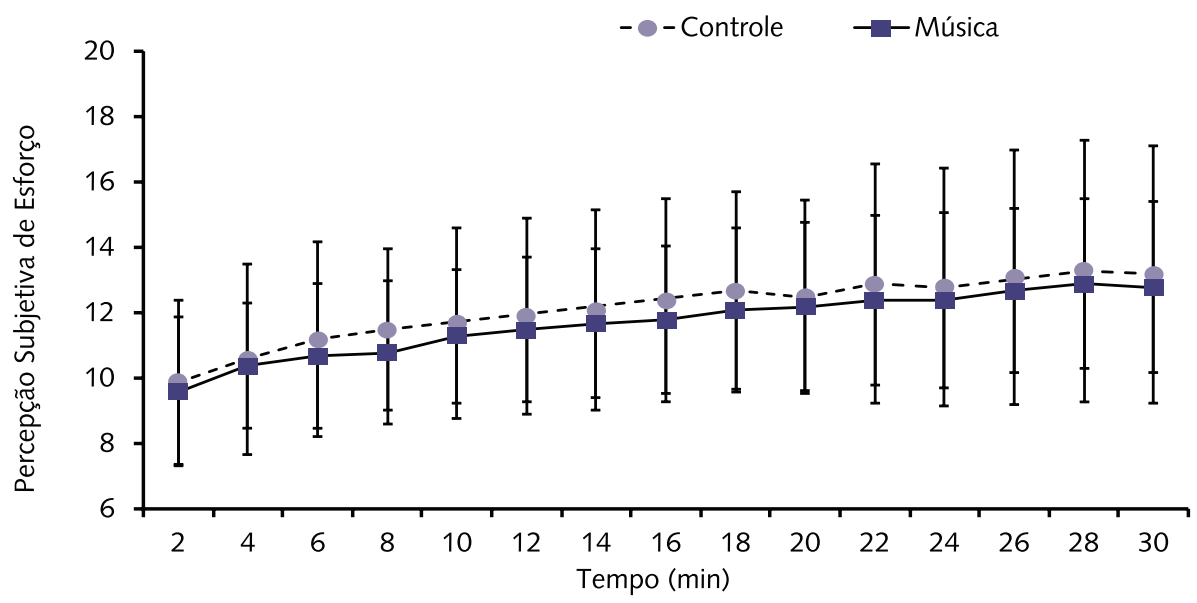

Figura 2 - Comportamento da percepção subjetiva de esforço a cada dois minutos (escala de Borg de 15 pontos) ao longo do teste de 30 minutos nas condições Controle e Música, expressa sob a forma média (marcadores) e desvio padrão (barra de erros). 
Com relação ao estado de humor dos sujeitos, somente no domínio fadiga ocorreu uma diferença significativa com uma pontuação maior ao final do teste do que no momento inicial $(P<0,05)$, conforme demonstrado na Figura 3, porém com mesmo comportamento sem diferença significativa $(P>0,05)$ entre as duas condições.

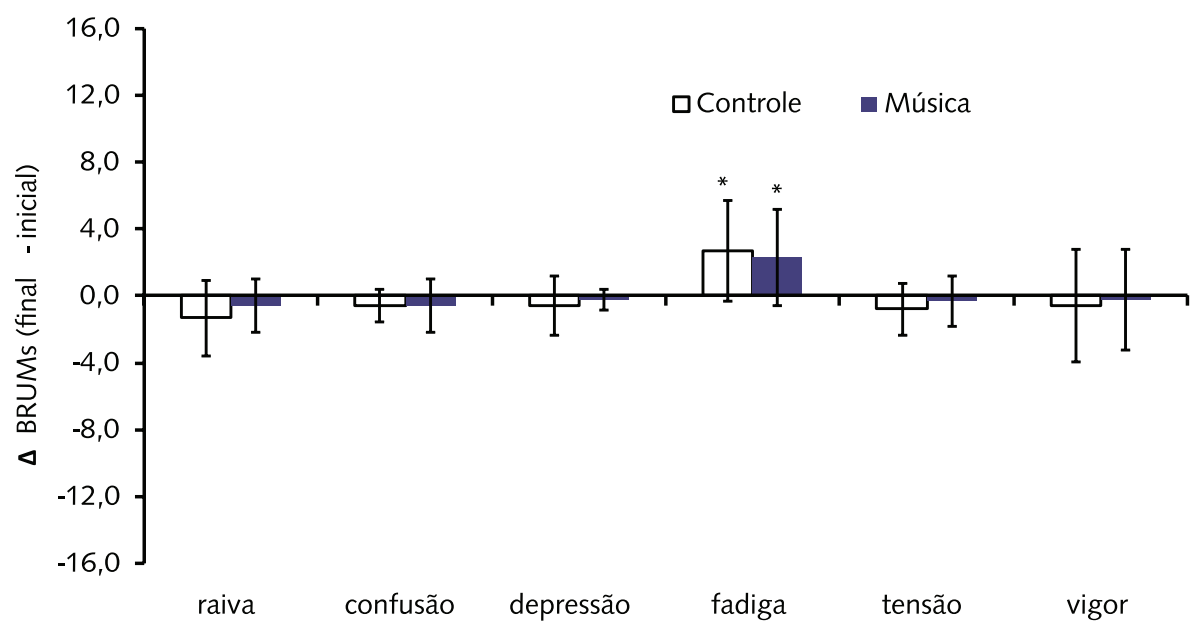

Figura 3 - Diferença entre a pontuação do final e inicial ( $\triangle$ BRUMS) do estado de humor dos voluntários nas condições Controle Música, expressa sob a forma média (colunas) e desvio padrão (barra de erros). * diferença significativa $(P<0,05)$ entre a pontuação de início e fim do domínio de humor na mesma condição.

A reativação parassimpática pós-exercício analisada através do $\mathrm{RMSSD}_{30 \mathrm{~s}}$ foi igual em ambas as condições (Figura 4).

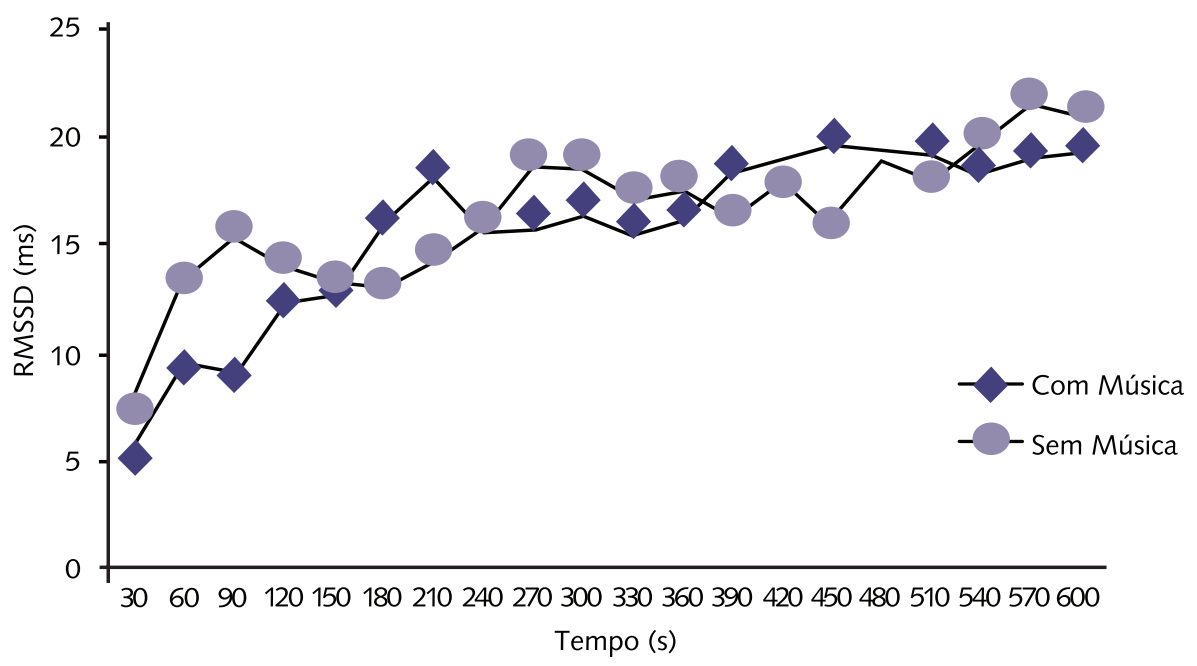

Figura 4 - Raiz quadrada da média das diferenças sucessivas ao quadrado entre intervalos R-R adjacentes, mensurado sobre sucessivos segmentos de 30s.

Quando comparado as mudanças relativas para os parâmetros da VFC entre as condições $\mathrm{M}$ e C, não houve diferença estatisticamente significante. Como esperado ambas as condições causaram perturbação autonômica (Figura 5). 


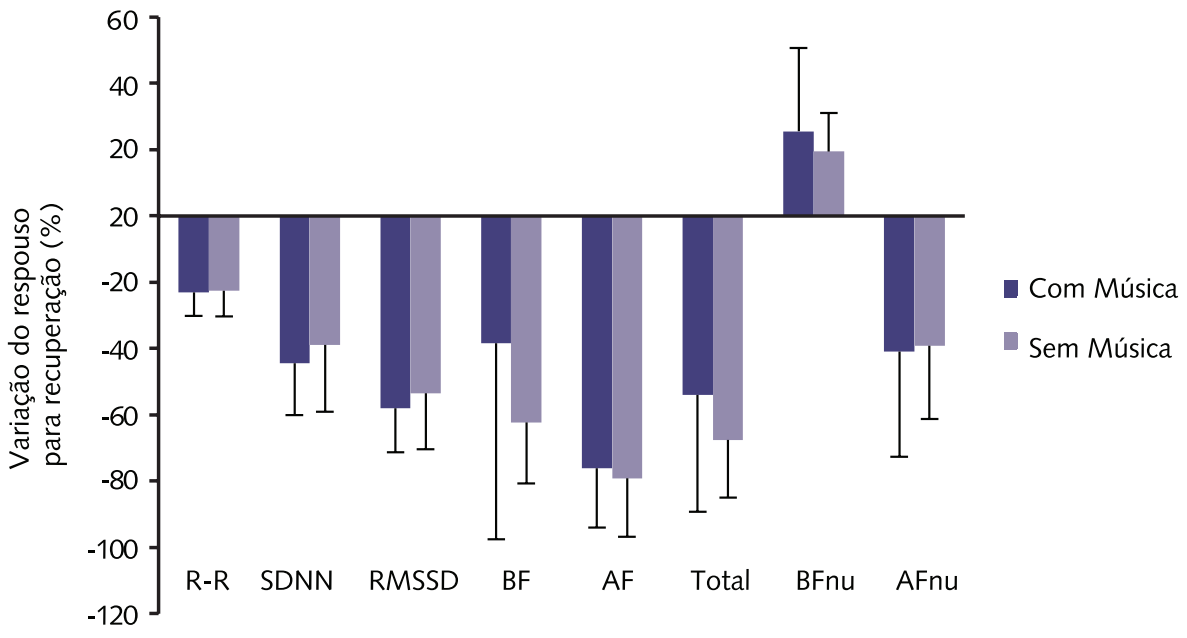

Figura 5 - Mudanças relativas dos parâmetros de VFC comparando repouso e recuperação nas condições música e controle. (Média \pm DP).

\section{DISCUSSÃO}

O objetivo deste estudo foi investigar se a música poderia influenciar variáveis psicológica e autonômicas durante e após uma sessão de exercício aeróbio de característica submáxima. Nesse caso não encontramos efeitos da música sobre qualquer variável psicológica ou autonômica.

As variações de potência ao longo do exercício foram similares entre as duas condições, sendo qualquer diferença explicada pela relação não totalmente direta dos sistemas perceptuais, fisiológicos e determinantes do desempenho, nesse caso, o exercício apresentou-se como um fator preponderante para os resultados de potência desenvolvida ao longo do exercício, quando um marcador de carga interna fora controlado. Além disso, a PSE e o EH não apresentaram qualquer diferença estatisticamente significante entre as condições experimentais, o que aponta que até mesmo a percepção do que se estava fazendo era similar, sendo o estímulo externo insuficiente para modular tal parâmetro, o que por sua vez poderia auxiliar nas variações de desempenho com ou sem o acompanhamento fisiológico.

Apesar da VFC já ter sido utilizada em estudo com protocolo de exercício semelhante ao nosso para verificar o efeito da música sobre este parâmetro, este foi o primeiro estudo a utilizar medidas de reativação parassimpática pós-exercício assim como o $\mathrm{RMSSD}_{30 \mathrm{~s}}{ }^{18}$, uma vez que acreditávamos que o estímulo externo carregado de componentes sensibilizadores pudesse ser capaz de alterar a PSE/potência ao longo da tarefa, modulando em subsequente o processo de recuperação apresentado pela VFC $\left(\mathrm{RMSSD}_{30}\right)$.

Desse modo, os resultados encontrados no presente estudo corroboram com os achados de um estudo prévio ${ }^{29}$, que demonstrou que o exercício atua de forma a provocar um estímulo maior do que a música para atividade do SNA. Nesse mesmo estudo, os autores demonstraram que índices espectrais da VFC foram afetados pela intensidade do exercício, mas não pela presença ou ausência de música. Além disso, os autores sugerem que o SNA pode ser afetado pelo estilo musical, o que não foi controlado no presente estudo podendo talvez explicar em parte a falta de diferença entre as condições.

Quando um recurso ergogênico psicológico é investigado os resultados podem aparecer muitas vezes mascarados pela subjetividade das informações e vari- 
áveis que são capazes de influenciar não só o desempenho e medidas fisiológicas, mas também respostas afetivas ao exercício. Como relatam alguns estudos que demonstraram uma série de modulações positivas no âmbito sensitivo, como o aumento do prazer pela realização do exercício, menor sensação de cansaço, melhor estado motivacional e melhor estado de ânimo ${ }^{5,7}$.

Waterhouse e colaboradores $(2009){ }^{6}$ verificaram uma interação entre o tempo da música e a faixa musical escolhida pelos participantes $(P<0,05)$, indicando resultados positivos nas variáveis: distância, potência e cadência, demonstrando resultados mais expressivos para as músicas rápidas $(3,8 \%, 9,8 \%$ e 5,9\%, respectivamente) quando comparadas as músicas lentas $(2,1 \%, 3,5 \%$ e $0,7 \%$, respectivamente). Os autores indicaram uma maior predisposição à realização dos exercícios submáximos propostos com a intervenção musical, assim como melhores resultados de desempenho dos participantes, atribuindo seus efeitos para hipóteses de funcionamento relacionadas ao humor e a distração.

Por outro lado Dyrlund e Wininger ${ }^{30}$ encontraram poucos resultados pelo uso de música em exercícios submáximos, demonstrando tímidas diferenças na percepção de esforço e ao foco de atenção, quando investigou os efeitos da música preferida e não preferida em uma grande amostra de 200 pessoas que realizaram 20 minutos de caminhada em diferentes intensidades de atividade. Em um estudo recente Bigliassi et al., ${ }^{11}$ encontraram um menor taxa de inclinação para um dos domínios do humor (fadiga) em corredores de 100 metros rasos após serem submetidos a uma estratégia psicológica prévia com musica e vídeo, variável esta que parece estar relacionada com o potencial de ativação prévio a uma determinada tarefa, diferente do modelo atualmente apresentado, no qual envolve um estratégia paralela ao exercício.

Dentro dessa perspectiva, tanto o nível de aptidão física dos sujeitos investigados corroborava com a situação previamente estipulada para que a música obteve-se efeito assim como a característica do exercício proposto, no caso de caráter submáximo, respeitando as recomendações para a prática de atividade física para indivíduos adultos pela CAME/AAC ${ }^{3}$. Por outro lado, algumas particularidades devem ser consideradas quanto ao aspecto não positivo dos efeitos da música nas medidas psicofisiológicas investigadas, como a escolha da própria música uma vez que essa possibilita melhor entendimento de como uma hipótese a partir de seus mecanismos de ação musical poderiam atuar durante o exercício, como por exemplo, seu o efeito sincronizador pelas batidas por minuto da música com a cadência de pedaladas na bicicleta. Nesse caso uma série de estudos apontam para tal efeito em diversas intensidades de exercício como forma de potencializar o desempenho dos participantes ou maximizar variáveis afetivas, quando estes são expostos a batidas rápidas, geralmente acima de 140 batimentos por minuto ${ }^{4,7,12}$.

Outro aspecto a ser considerado diz respeito ao não controle do volume no qual a música foi aplicada, uma vez que à medida que estímulos externos auditivos detêm maior volume em decibéis, nos quais esses tendem a ser interpretados com maior freqüência, principalmente em exercícios submáximos onde a resposta muscular por estímulos aferentes não apresenta tamanha prevalência sobre estímulos externos como é o caso de exercícios máximos, ou dependentes de um alto nível de concentração na tarefa ${ }^{10,30}$.

Por fim, apesar das limitações descritas anteriormente as quais entendemos ser de difícil controle, uma vez que depende do gosto musical bem como da percepção individual de cada sujeito e da resposta individual de cada sujeito para com estímulos auditivos externos, concluímos que a música escolhida pelos próprios 
sujeitos não foi capaz de modular variáveis psicofisiológicas em uma atividade submáxima em ciclossimulador.

\section{Agradecimentos}

Os autores agradecem aos Professores Marcelo Vitor da Costa e José Luiz Dantas que contribuíram na coleta e análise dos dados, bem como ao CNPq, CAPES e Fundação Araucária pelo apoio financeiro e bolsas outorgadas.

\section{REFERÊNCIAS}

1. Nahas MV. Atividade física saúde e qualidade de vida: Londrina: Midiograf, 2001.

2. Reichert FF, Barros AJ, Domingues MR, Hallal PC. The role of perceived personal barriers to engagement in leisure-time physical activity. Am J Public Health 2007;97:515-9

3. Haskell WL, Lee IM, Pate RR, et al. Physical activity and public health: updated recommendation for adults from the American College of Sports Medicine and the American Heart Association. Med Sci Sports Exerc 2007;39:1423-34.

4. Karageorghis CI, Terry P. The psychophysical effects of music in sport and exercise: a review. J Sport Behav 1997;20:54-68.

5. Elliott D, Carr S, Savage D. Effects of motivational music on work output and affective responses during sub-maximal cycling of a standardized perceived intensity. J Sport Behav 2004;27:134-47.

6. Waterhouse J, Hudson P, Edwards B. Effects of music tempo upon submaximal cycling performance. Scand J Med Sci Sports 2009;20:662-69.

7. Karageorghis CI, Mouzourides DA, Priest DL, et al. Psychophysical and ergogenic effects of synchronous music during treadmill walking. J Sport Exerc Psychol. 2009;31:18-36.

8. Nakamura PM, Pereira G, Papini CB, Nakamura FY, Kokubun E. Effects of preferred and nonpreferred music on continuous cycling exercise performance. Percept Motor Skills 2010;110:257-64.

9. Szmedra L, Bacharach DW. Effect of music on perceived exertion, plasma lactate, norepinephrine and cardiovascular hemodynamics during treadmill running. Int J Sports Med 1998;19:32-37.

10. Bigliassi MB, Dantas JL, Carneiro JG, Smirmaul BPC, Altimari LR. Influence of music and its moments of application on performance and psychophysiological parameters during a $5 \mathrm{~km}$ time trial. Rev Andal Med Deporte 2012;5:83-90.

11. Bigliassi MB, Kanthack TFD, Carneiro JG, Seron BB, Dourado AC, Altimari LR. Intervenção psicológica prévia: Efeito de uma estratégia sensorial nos $100 \mathrm{~m}$ rasos. Braz J Biomotricity 2012;6:203-212.

12. Carneiro JG, Bigliassi M, Dantas JL, Souza SR, Altimari LR. Música: Recurso ergogênico psicológico duranto o exercício físico. Rev Bras Psicol Esporte 2010;3:61-70.

13. Tanaka H, Monahan KD, Seals DR. Age-predicted maximal heart rate revisited. J Am Coll Cardiol. 2001;37:153-6.

14. Machado FA, Denadai BS. Validade das equações preditivas da frequência cardíaca máxima para crianças e adolescentes. Arq Bras Cardiol 2011;97:136-140.

15. Barbosa FP, Oliveira HB, Fernandes PR, Fernandes FJ. Estudo Comparativo de Equações de Estimativa da FC Máxima. Fit Perf J 2004;3:108-114.

16. Camarda SRA, Tebxreni AS, Páfaro CN, Nasai FB, Tambeiro VL, Juliano Y, Neto TLB. Comparação da Freqüência Cardíaca Máxima Medida com as Fórmulas de Predição Propostas por Karvonen e Tanaka. Arq Bras Cardiol 2008;91;311-314.

17. Nakamura PM, Deustch S, Kokubun E. Influence of preferred and non-preferred music on the mood and perfomance during heavy intensity exercise. Rev Bras Educ Fís Esporte 2008;22:247-55.

18. Task Force of the European Society of Cardiology and the North American Society of Pacing and Electrophysiology. Heart rate variability: standards of measurement, physiological interpretation and clinical use. Circulation 1996;93:1043-65.

19. Guzik P, Piskorski J, Krauze T, et al. Correlations between the Poincare plot and conventional heart rate variability parameters assessed during paced breathing. J Physiol Sci.2007;57:63-71. 
20. Gamelin FX, Berthoin S, Bosquet L. Validity of the polar S810 heart rate monitor to measure R-R intervals at rest. Med Sci Sports Exerc 2006;38:887-93.

21. Terry PC, Lane AM, Fogarty GJ. Construct validity of the Profile of Mood States -Adolescents for use with adults. Psychol Sport Exerc 2003;4:125-39.

22. Anglem N, Lucas SJ, Rose EA, Cotter JD. Mood, illness and injury responses and recovery with adventure racing. Wilderness Environ Med 2008;19:30-8.

23. Rohlfs ICPM. Validação do Teste Brums para Avaliação de Humor em Atletas e Não Atletas Brasileiros. Florianópolis: Universidade Estadual de Santa Catarina, 2006.

24. Rohlfs ICPM, Rotta TM, Luft CB, Andrade A, Krebs RJ, Carvalho T. A Escala de Humor de Brunel (Brums): instrumento para detecção precoce da síndrome do excesso de treinamento. Rev Bras de Med Esporte 2008;14: 176-81.

25. Borg GA. Psychophysical bases of perceived exertion. Med Sci Sports Exerc 1982;14: 377-81.

26. Borg GA. Perceived exertion as an indicator of somatic stress. Scand J Rehabil Med 1970;2:92-8.

27. Garcin M, Fleury A, Mille-Hamard L, Billat V. Sex-related differences in ratings of perceived exertion and estimated time limit. Int J Sports Med. 2005;26:675-81.

28. Marcora SM, Staiano W. The limit to exercise tolerance in humans: mind over muscle? Eur J Appl Physiol. 2010;109:763 -70.

29. Yamashita S, Iwai K, Akimoto T, Sugawara J, Kono I. Effects of music during exercise on RPE, heart rate and the autonomic nervous system. JSports Med Phys Fitness 2006;46: 425-30.

30. Dyrlund AK, Wininger SR. The effects of music preference and exercise intensity on psychological variables. J Music Ther. 2008;45:114-34.



